Review

\title{
Cultural versus biological inheritance: A retrospective view of Cavalli-Sforza and Feldman (1973)
}

Hao Shen and Marcus W. Feldman*

\author{
Department of Biology, Stanford University, Stanford, CA 94305-5020, USA; \\ E-Mail: shenhao@stanford.edu
}

* Correspondence: Marcus W. Feldman; E-Mail: mfeldman@stanford.edu

Received: 16 Apr 2021

Accepted: 18 Jun 2021

Published: 16 Oct 2021

\section{Copyright:}

(C) 2021 by the author(s).

This is an Open Access

article distributed under the terms of the Creative

Commons License

Attribution 4.0

International (CC BY 4.0),

which permits unrestricted

use, distribution, and

reproduction in any

medium or format,

provided the original work

is correctly credited.

\section{Publisher's Note:}

Pivot Science Publication

Corp. remains neutral with regard to jurisdictional claims in published maps and institutional affiliations.

\begin{abstract}
The early 1970s was a period of ferment in human population genetics that resulted from the insistence of Arthur Jensen and William Shockley that intelligence was genetically determined, and that public policy should reflect this. They claimed that high heritability within a population indicated genetic differences between populations. The heritability statistic played a major role in the debate that ensued. The mathematical and computational analysis in Cavalli-Sforza and Feldman (1973) showed that the direct phenotypic transmission from parents to children could produce the appearance of high heritability even though the transmission was not genetic. This paper stimulated decades of statistical reanalysis of data on intelligence and other quantitative phenotypes in which the roles of cultural transmission and assortative mating were shown to be fundamental. Research into direct and indirect effects, causation and confounding, and gene-culture coevolution, carried out over the subsequent decades, can be traced to the framework established by Cavalli-Sforza and Feldman (1973).
\end{abstract}

Based on "Cavalli-Sforza LL, Feldman MW. Cultural versus biological inheritance: Phenotypic transmission from parents to children (A theory of the effect of parental phenotypes on children's phenotypes). Am. J. Hum. Genet. 1973; 25:618-637."

Keywords: heritability; cultural transmission; correlations between relatives; direct and indirect effects; gene-culture coevolution

\section{Introduction}

Sewall Wright's application of path analysis $[1,2]$ to the data on IQ of biological and adopted children collected by Barbara Burks [3] was one of the earliest attempts to partition intelligence into genetic (Wright called it "heredity") and 
environmental components. Wright's careful language and frequent caveats in interpreting his conclusions were not emulated by those who decades later applied his path analysis methods to larger data sets on IQs of different relatives.

Almost forty years after Wright's path analysis of IQ, the educational psychologist, Arthur Jensen, focused on the statistic "heritability" as a measure of the extent of genetic determination of IQ, stating that "heritability tells us the correlation between genotypes and phenotypes in the population" [4]. He described as the "most satisfactory" (p. 478) and "most interesting" (p. 52) estimate of the heritability of IQ, that of Burt [5], namely 0.86 . Burt's analysis was discredited by Kamin [6] as being based on fraudulent data, and Kamin's 1974 book [7] critically examined all of the data from studies of twins reared together and apart up to that time and provided an excellent empirical context in which to assess Jensen's claims.

The reductionist ferment that followed the publication by Arthur Jensen in 1969 in the Harvard Educational Review of his article "How Much Can We Boost IQ and Scholastic Achievement?" [4] was amplified at Stanford (and, in fact, across the U.S.A.) by the profoundly racist pronouncements of Stanford Professor of Engineering, William Shockley. Both Jensen and Shockley expounded the view that intelligence was primarily genetically determined and that the inferior measured intelligence and school performance of African-Americans compared to white Americans was a result of genetic differences between the two groups. For both Jensen and Shockley, the key statistic they used to justify their conclusions was the heritability of measured IQ, which had been estimated in studies in the U.K. in which white monozygous twins reared together were compared with those reared apart. Jensen and Shockley viewed the published value of $80 \%$ and $90 \%$ heritability as proof of the genetic determination of IQ and a justification of their claim that the observed difference in intelligence between African and European Americans was due to genetics.

Most geneticists were appalled by the naked racism that was espoused by Jensen and Shockley in their very public pronouncements on racial differences in intelligence and on the misuse of the heritability statistic to justify their racist claims. Luca Cavalli-Sforza, who had recently moved from Italy to become professor of genetics at Stanford, was profoundly disturbed by what he perceived as the potential for the public, and potentially other scholars, to be influenced by Jensen and Shockley, who had acquired significant access to mass media. 
One of us (MWF) had also recently arrived at Stanford, and in 1972 CavalliSforza and MWF began discussing the potential damage being done by Jensen and Shockley, and, in particular, what inferences could be drawn from the heritability statistic that was such an integral part of their speeches. These discussions gave rise to our mathematical work on cultural transmission and gene-culture interactions, a collaboration that lasted forty years.

The starting point for this collaboration centered on an attempt to analyze how correlations between relatives for a behavioral trait could be affected by cultural transmission of that trait (or other traits) from parents (which we called "vertical transmission") or others of the parental generation ("oblique transmission") or of one's own cohort ("horizontal transmission"). Correlations between relatives (at that time, and still often today) were the raw material from which the heritability statistic was computed. Our original goal was to explore how heritability would be affected by cultural transmission; and, since parents obviously constitute the major part of a child's environment, phenotypic transmission from parents could confound inferences about the importance of genes in phenotypic determination.

This project led to our 1973 publication [8] (referred to here as " $\mathrm{C}$ and F") and raised several statistical and philosophical issues that remain central to the biological sciences today. First, heritability is a statistic usually defined as the fraction of phenotypic variation associated with differences among genotypes. This definition is not complete unless there is an underlying model for how genes, phenotypes, and environments of parents and others might contribute to an individual's phenotype and hence how the phenotypic variance should be decomposed into components. The second issue concerned what kinds of data are appropriate for estimating these components. Third, does heritability or its estimate have a role in assessing the cause of a phenotype and can such causes be identified? A final question concerns the purpose to which any estimate of heritability could or should be used. These issues will be discussed below as we explore some of the ramifications of $C$ and $F$.

$\mathrm{C}$ and $\mathrm{F}$ wrote a general model for the phenotype, $\phi_{i m}$, of a child of genotype $\mathrm{i}$, as

$$
\phi_{i m}=f_{i}\left(\phi_{F}, \phi_{M}\right)+\varepsilon
$$


where $\phi_{F}$ and $\phi_{M}$ are the father's and mother's phenotypes and $\varepsilon$ is a random, non-familial variable with mean zero and variance $\sigma^{2}$. A particular case of eq. (1) that allows detailed analysis is

$$
\phi_{i m}=a_{i}+2 b_{i} \phi_{m}+\varepsilon
$$

where $a_{i}$ is the independent effect of the offspring's genotype, and $b_{i}$ controls the extent to which parental phenotypes affect the child with genotype $i$. Here $\phi_{m}$ is the mean parental phenotype, and $\varepsilon$ is, as before, a random non-familial variable. For the model in Eq. (2), C and F calculated the total phenotypic variance at equilibrium and the variance within families at equilibrium, as well as correlations between various relatives. As we shall see below, the meaning of the "genetic variance", which is central to the calculation of heritability, is a vexing question, as is its appropriate estimation.

The paper raised several points concerning the estimation and interpretation of the heritability statistic that remain relevant even in today's era of genome-wide association studies (GWAS). First, their formulation in Eq. (2) makes explicit the relevance of the norm of reaction through the role of the parameter, $b_{i}$, which measures how a child's genotype, $i$, responds to different parental phenotypic pairs, which constitute at least part of its environment. Second, they point out that their idealized framework does not take account how traits other than parents' IQs might influence an offspring's IQ. Third, they raise the issue, discussed in more detail below, of the different measures that are assigned the name 'heritability', in both a broad and narrow sense. A fourth point is that in the setting of cultural transmission, equilibrium is not reached immediately, as it is in the purely genetic treatments based on the famous paper by Fisher [9]. Feldman et al. [10] showed that the frequencies of alleles at the Hardy-Weinberg equilibrium, assumed in almost all analyses of correlations between relatives, can have important effects on these correlations and hence on heritability measures. If the frequencies of the phenotypes $\phi$ in Eq. (2) change over time, so will the covariances between relatives that produce the heritability statistics. Dual inheritance (cultural and genetic) can give rise to a kind of gene-environment correlation that can cause serious problems in analysis and interpretation of the variance decomposition. We expand on these points below.

In the decade subsequent to $\mathrm{C}$ and $\mathrm{F}$, a number of analyses of familial data related to IQ and other traits were published, many of which returned to Wright's original path analysis framework. Newton Morton's group at the 
University of Hawaii used these methods on a data set of familial correlations, including those of Burt, and estimated the heritability of IQ to be 0.75 [11]. Later, this group revised their estimate to be 0.67 [12].

These analyses by Rao et al. did not include cultural transmission, which was, however, incorporated into the statistical treatments by Cloninger et al. $[13,14]$. Their models incorporated both cultural transmission and assortative mating and produced estimates of heritability of IQ close to 0.3. In 1982, Morton's group again used path analysis on a larger data set than in their earlier papers, but this time they estimated cultural heritability to be substantially higher than their estimate of genetic heritability [15]. All of these studies incorporating statistical analysis of cultural transmission should be regarded as having been stimulated by the models first introduced in 1973 by $\mathrm{C}$ and $\mathrm{F}$.

C and F (1973) was the first in their series of studies of continuously varying phenotypes, in which they incorporated mutation and stabilizing selection [16]. In addition to phenotypic transmission, they extended their model to include various kinds of assortative mating [17-19]. A general summary of these studies and those of Morton's group, focusing on the role of cultural transmission and different aspects of assortative mating, as well as estimation techniques, can be found in Otto et al. [20].

The phenogenotype models described by Eqs. (1) and (2) refer to continuously varying (vertically) transmitted traits. However, the spirit of these models can be adapted to discrete valued traits that are culturally transmitted and for which there is assortative mating. Extensions to include selection on discrete valued culturally transmitted phenotypes have also been made $[21,22]$. Some of the evolutionary models that incorporate assortative mating for and various modes of cultural transmission of such discrete traits are the direct descendants of $\mathrm{C}$ and $\mathrm{F}$ and are summarized in the next section.

\section{A Discrete Bilinear Version of Cavalli-Sforza and Feldman (1973)}

An individual's combined genotype and phenotype is called its 'phenogenotype'. For a dichotomous phenotype, for example bar and not bar, and for a diploid diallelic genetic locus with alleles $A_{1}$ and $A_{2}$, we can write a version of Eq. (2) using Table 1, below [10,23], where the entries are the probabilities that the genotype in that column with parents having phenotypes in that row have the bar phenotype. Thus the entries give the probabilities of a child's phenogenotype, where $\varepsilon_{1}, \varepsilon_{2}, \varepsilon_{3}$ are the contributions from the child's 
genotype, $A_{1} A_{1}, A_{1} A_{2}, A_{2} A_{2}$, respectively; $a_{1}, a_{2}, a_{3}$ are contributions from a bar parent to offspring of genotype $A_{1} A_{1}, A_{1} A_{2}, A_{2} A_{2}$, respectively, and $b_{1}, b_{2}, b_{3}$ are the corresponding contributions from a non-bar parent.

Table 1 Transmission table. The first row shows the child's genotype; the first column represents parents' pheno-genotypes, and the entries represent the child's probability of having the bar phenotype conditioned on the child's genotype (first row) and the parents' phenotypes (first column).

\begin{tabular}{|c|c|c|c|}
\hline & $A_{1} A_{1}$ & $A_{1} A_{2}$ & $A_{2} A_{2}$ \\
\hline$\overline{A_{i} A_{j}} \times \overline{A_{k} A_{h}}$ & $\varepsilon_{1}+2 a_{1}$ & $\varepsilon_{2}+2 a_{2}$ & $\varepsilon_{3}+2 a_{3}$ \\
\hline$\overline{A_{i} A_{j}} \times A_{k} A_{h}$ & $\varepsilon_{1}+a_{1}+b_{1}$ & $\varepsilon_{2}+a_{2}+b_{2}$ & $\varepsilon_{3}+a_{3}+b_{3}$ \\
\hline$A_{i} A_{j} \times \overline{A_{k} A_{h}}$ & $\varepsilon_{1}+a_{1}+b_{1}$ & $\varepsilon_{2}+a_{2}+b_{2}$ & $\varepsilon_{3}+a_{3}+b_{3}$ \\
\hline$A_{i} A_{j} \times A_{k} A_{h}$ & $\varepsilon_{1}+2 b_{1}$ & $\varepsilon_{2}+2 b_{2}$ & $\varepsilon_{3}+2 b_{3}$ \\
\hline
\end{tabular}

The phenogenotypic transmissions in Table 1, from Shen and Feldman (2020), allow explicit calculation of correlations between relatives $[10,23]$. This analysis can be extended to include assortative mating or population subdivision [23]. It also explicitly raises the question of how the various covariance calculations can give rise to various notions of heritability and inferences about causality.

In the context of GWAS, a model closely related to that of $\mathrm{C}$ and $\mathrm{F}$ was proposed by Kong et al. [24] (see also Young et al. [25]), who found that parent's nontransmitted alleles could be correlated with their offspring's phenotypes. They suggested that this correlation could arise from the effects of the parents' genotypes on their phenotypes which, as in C and F, could be culturally transmitted (vertical transmission) to the offspring. Shen and Feldman [23] proposed a unified causal framework for this phenomenon, which Kong et al. called "genetic nurturing" [24].

Here we introduce the theoretical framework that forms a background to historical developments related to $C$ and $F$. The definition, meaning, and estimates of heritability will be introduced and the effect of different kinds of cultural transmission will be discussed.

\section{Phenotypic Decompositions and Heritability}

In statistical genetics, there are two types of heritability of a measured phenotype: broad sense heritability, which we refer to as $H_{\text {broad }}^{2}$ and narrow sense heritability, which we refer to as $H_{\text {narrow }}^{2}$. The definitions of these two types of heritability are related to the decomposition of total phenotypic variance, which, in turn, emerges from a decomposition of the phenotypic value, and was originally framed as a partition of the phenotype's measured 
value into its potential causes. Here we give a simple introduction to this process.

A phenotypic value, $P$, is first decomposed into a genetic component and an environmental component, i.e., $P=E\left(P \mid S_{G}\right)+e$, where $S_{G}$ is the space of measurable functions of the genotype (represented by numbers of alleles different at loci), $E\left(P \mid S_{G}\right)$ is the genetic component, and $e$ is the nontransmitted environmental component (also known as the 'residual'). Because $E\left(P \mid S_{G}\right)$ is the projection of $P$ onto $S_{G}, E\left(P \mid S_{G}\right)$ is orthogonal to $e$ and accordingly, allows the variance decomposition $V=V_{G}+V_{E}$, where $V=\operatorname{Var}(P)$ is the total phenotypic variance; $V_{G}=\operatorname{Var}\left(E\left(P \mid S_{G}\right)\right)$ is the genetic variance; $V_{E}=\operatorname{Var}(e)$ is the environmental variance. The broad sense heritability is then defined as $H_{\text {broad }}^{2}=\frac{V_{G}}{V}$, i.e., genetic variance as a fraction of total variance.

This decomposition process can be extended. Let $S_{L}$ be the space of functions that are sums of measurable functions of allele numbers of every locus, and $S_{A}$ the space of linear functions of allele numbers. Then we have $E\left(P \mid S_{G}\right)=E\left(P \mid S_{L}\right)+i$, where $i$ is called the 'epistatic' component, and $E\left(P \mid S_{L}\right)=E\left(P \mid S_{A}\right)+d$, where $E\left(P \mid S_{A}\right)$ is the additive component and $d$ is called the 'dominance' component. Because $S_{L}$ is a subspace of $S_{G}$, and $S_{A}$ is a subspace of $S_{L}$, we know that $E\left(P \mid S_{A}\right), d$, and $i$ are orthogonal to each other. The variance decomposition then becomes $V_{G}=V_{A}+V_{I}+V_{D}$, where $V_{A}=\operatorname{Var}\left(E\left(P \mid S_{A}\right)\right)$ is the additive genetic variance, $V_{I}=\operatorname{Var}(i)$ is the epistatic variance and $V_{D}=\operatorname{Var}(d)$ is the dominance variance. The narrow sense heritability is then defined as $H_{\text {narrow }}^{2}=\frac{V_{A}}{V}$.

The additivity in $V_{A}$ stands for both additivity among loci, namely the additive effect of different alleles at different loci, and within locus additivity, i.e., the additive effect of different alleles within a locus. Also note that there is no specific name for $E\left(P \mid S_{L}\right)$ and $\operatorname{Var}\left(E\left(P \mid S_{L}\right)\right)$, which only represent additivity among loci. In principle we might also call $\frac{\operatorname{Var}\left(E\left(P \mid S_{L}\right)\right)}{V}$ the intermediate sense heritability, $H_{\text {intermediate, }}^{2}$ because it is between broad sense heritability and narrow sense heritability. However, this is not used because the effect of epistasis is usually ignored, either because researchers believe it is minor or because it causes serious statistical difficulties. We will mention one of the problems below, and a more detailed analysis is given in the discussion.

The above decompositions of the phenotypic value and the total phenotypic variance can always be made in principle, given the availability of data describing the distribution of phenogenotypes. However, the decomposition raises some interesting issues. First, why estimate the broad or narrow 
sense heritabilities? What do we want to know about heritability, or want it to represent? Second, what do these definitions of heritability actually mean? Can they really represent the quantities we want them to represent? Third, in general we do not have appropriate data about the distribution of phenogenotypes, so how do we estimate heritability? Finally, are these estimates biased?

Three kinds of issues are involved here: the quantity we want 'heritability' to represent, the definition of heritability, and the estimate of heritability. For the quantity heritability is supposed to represent, we want to know whether it is meaningful under different situations. For the different definitions of heritability, we want to know whether they really represent the quantity we want them to represent, and we want to know whether the estimates arising from different definitions are unbiased.

First, what should heritability represent? The original purpose of heritability was to estimate the fraction of variance (deviation from the mean) 'caused' (more often people use 'explained', but this term has a subtext that involves causation) by genetic differences. We have already introduced two definitions of heritability, the narrow sense and broad sense, and later we will introduce a third. Finally, how do we estimate heritability empirically? Before the genomic era, pedigree analysis was used to estimate narrow sense and broad sense heritabilities. In the genomic era, phenotypes are regressed on features of single nucleotide polymorphisms (SNPs) to estimate narrow heritability directly according to the DNA sequence.

In traditional statistical genetics, these three quantities coincide with each other under very simple assumptions, i.e., a purely additive model with random mating. To be more specific, for each phenotypic value, $P$, assume that $P=A+E$, where $A$ is the genetic component, a linear function of allele numbers, which is what 'additive' stands for (no epistasis and dominance), and $E$ is an environmental component, independent of the genotype (implying that it is also independent of $A$.) Then for the genetic/additive component corresponding to the broad sense and narrow sense heritabilities, $E\left(P \mid S_{G}\right)=$ $E\left(P \mid S_{A}\right)=A$. There is no dominance or epistatic variance since additivity is assumed. All estimates of heritability based on pedigrees should produce the same value, i.e., $\frac{\operatorname{Var}(A)}{V}$. Because it is assumed that there is no dominance and epistasis, GWAS should also return the same quantity. In this model, the only active path from the genotype to the phenotype is a causal one, represented by $A$. Thus, the three kinds of quantities should coincide with each other. 
The purely additive model can be extended slightly by adding dominance variance (but not epistasis variance), in which case there will also be only one path from genotype to phenotype, and it is causal. Thus, the equivalence between the fraction of variance 'caused' by genetic differences and broad sense heritability will still hold. However, in this case, the broad sense heritability and narrow sense heritability will no longer be the same. Also, the different pedigree estimates will not be the same. However, this is not a big problem because the broad sense heritability may still represent the desired quantity, namely the fraction of variance that is caused by genetic differences. A slightly more complicated pedigree analysis can produce an estimate of broad sense heritability, and this can also be done in principle for GWAS.

Inclusion of epistasis makes it very hard, if not impossible to use pedigree analysis or GWA to estimate broad sense heritability. However, broad sense heritability can still be defined with the desired meaning, since again, in the underlying model, the only active path from the genotype to the phenotype is a causal one. We will come back to the problem of epistasis in the discussion.

Things become more complicated when we try to do the same thing in a more realistic setting. To see this, recall what heritability is supposed to represent: an estimate of the fraction of variance 'caused' by genetic differences. Notice that we put 'caused' in quotes, because it is well known that correlation does not mean causation. In a more realistic setting there might be multiple active paths from the genotype to the phenotype, and some might be non-causal. Although it is defined in terms of the phenotypic variance among different genotypes, we can not expect 'heritability', especially broad sense heritability, to faithfully represent variance that is 'caused' by genetic differences, as in the above toy models. Also, under these settings, we can not guarantee that the estimate of heritability from pedigree analysis is unbiased. Most importantly, even if we can get an unbiased estimate of the quantity we want heritability to represent, i.e., the fraction of variance 'caused' by genetic differences, it may be not meaningful if, as explained in the following paragraphs, the variance cannot be decomposed.

\section{Direct Cultural Transmission}

These problems can best be illustrated in the case of direct cultural transmission, as first introduced by $\mathrm{C}$ and $\mathrm{F}$. Here we start by introducing the basic model of cultural transmission for a continuously varying phenotype. 
For such a trait, assume $P=A+B+E$ (after normalization, i.e., subtracting the mean), where $A$ is the genetic component, which may be assumed to be additive, i.e., $A_{O}=\frac{1}{2}\left(A_{F}+A_{M}\right)$, with $A_{F}$ and $A_{M}$ representing the father's and mother's additive genetic values. $B$ is the cultural component, and $E$ is the environmental component, which is assumed to be an independent variable for each individual. We say the transmission is direct if the value of an individual's cultural component is a function of its parents' phenotypic values, i.e., $B_{O}=f\left(P_{F}, P_{M}\right)$, and in the linear case, $B_{O}=\beta_{D}\left(P_{F}+P_{M}\right)+\delta_{D}$, where $\delta_{D}$ is a random variable that represents transmission error, and $\beta_{D}$ is a transmission coefficient. We call the transmission indirect if the cultural component is a function of one's parents' cultural values, i.e., $B_{O}=f\left(B_{F}, B_{M}\right)$, and for the linear case we can similarly assume $B_{O}=\beta_{I}\left(B_{F}+B_{M}\right)+\delta_{I}$.

Now assume random mating, no genetic epistasis, independence between $E$ and $A, B$, in the linear model of direct cultural transmission shown above. All the problems we mentioned above are recapitulated. First, the quantity we want heritability to represent, the fraction of variance 'caused' by genetic differences is $\frac{\operatorname{Var}(A)}{V}$. However, it is important to note that $A$ here is neither $E\left(P \mid S_{G}\right)$ nor $E\left(P \mid S_{A}\right)$. This is because $A$ and $B+E$ are correlated, i.e., $\operatorname{Cov}\left(A_{O}, B_{O}+E_{O}\right)=$ $\operatorname{Cov}\left(A_{O}, B_{O}\right)=\frac{\beta_{D}}{2} \operatorname{Cov}\left(A_{F}+A_{M}, P_{F}+P_{M}\right)=\beta_{D} \operatorname{Cov}(A, P) \neq 0$; here $\operatorname{Cov}(A, P)$ represents the covariance of genetic value and phenotypic value, and in general cannot be assumed to be close to zero. This means $A$ can not be $P$ 's projection onto any space, neither $E\left(P \mid S_{G}\right)$ nor $E\left(P \mid S_{A}\right)$. As a consequence, it is not surprising that $\frac{\operatorname{Var}(A)}{V}$ is neither the broad sense heritability nor the narrow sense heritability. This can easily be shown through direct calculation (which we do not include here). A common mistake in research that takes direct cultural transmission into account is to assume that $\frac{\operatorname{Var}(A)}{V}$ is the narrow sense or broad sense heritability. In order to distinguish this quantity from $H_{\text {broad }}^{2}$ and $H_{\text {narrow, }}^{2}$ we represent it by $h^{2}$. Second, the estimate from pedigree analysis will be biased. Take the most common estimate, for example, $2\left(r_{M Z}-r_{D Z}\right)$, where $r_{M Z}$ and $r_{D Z}$ are the phenotypic correlations between monozygous and dizygous twins, respectively; this gives us $\frac{\operatorname{Var}(A)}{V}$ instead of broad or narrow sense heritability. The $\mathrm{MZ}$ covariance will be $\operatorname{Var}(A)+\operatorname{Var}(B)+2 \operatorname{Cov}(A, B)$, while the DZ covariance is $\frac{\operatorname{Var}(A)}{2}+\operatorname{Var}(B)+2 \operatorname{Cov}(A, B)$. This might be regarded as an acceptable mistake; although $\frac{\operatorname{Var}(A)}{V}$ is a biased estimate of $H_{\text {broad }}^{2}$ or $H_{\text {narrow, }}^{2}$ it gives us the quantity we want heritability to represent, namely, the fraction of variance caused by genetic differences.

Unfortunately, this mistake is not acceptable, because the quantity we want heritability to represent is not meaningful in the first place! Why? Because 
when we say a fraction of variance is 'explained' or 'caused' by something, it is only meaningful when a clean decomposition of total variance is possible, i.e., a decomposition without a covariance term. However, as we have shown, $\operatorname{Cov}(A, B) \neq 0$, so we do not have a clean decomposition. The total variance will be $\operatorname{Var}(A)+\operatorname{Var}(B)+2 \operatorname{Cov}(A, B)+\operatorname{Var}(E)$. Since $\operatorname{Cov}(A, B)$ could in principle be negative, the quantity $h^{2}=\frac{\operatorname{Var}(A)}{V}$ might even be greater than 1 in extreme cases and thus would be meaningless. Some people might say that this is because $h^{2}$ does not represent the broad sense heritability or the narrow sense heritability, and if we estimate the broad or narrow sense properly, we will achieve a clean decomposition of the total phenotypic variance. This is true, of course, but what does the broad/narrow sense heritability mean, given that it does not represent the fraction of variance 'caused' by genetic differences? Actually $E\left(P \mid S_{G}\right)$ and $E\left(P \mid S_{A}\right)$ will be a mixture of genetic and cultural components. Thus, under this situation, $H_{\text {broad }}^{2} H_{\text {narrow }}^{2} h^{2}$ and the pedigree estimates are all meaningless in any causal sense. In this situation the only meaningful variance decomposition will be $V=\operatorname{Var}(A+B)+\operatorname{Var}(E)$, which requires that we do not distinguish between genetic transmission and direct cultural transmission.

For indirect cultural transmission, it depends on whether $A$ and $B$ are correlated. If they are correlated, i.e., $\operatorname{Cov}(A, B) \neq 0$, it will be similar to the direct cultural transmission case and all the same problems will occur. If $A$ and $B$ are assumed not to be correlated, then $H_{\text {broad }}^{2}, H_{\text {narrow }}^{2} h^{2}$ and $2\left(r_{M Z}-r_{D Z}\right)$ will all be the same.

In the widely used $A C E$ model, which decomposes $P$ into $A$, the genetic component, $C$ the common environmental component, and $E$ the individual non-transmitted environmental component, it is easy to see that under cultural transmission, $B$ from the previous paragraph will be the common environment. In a typical $A C E$ model, $A$ and $C$ are assumed to be independent; the corresponding model with cultural transmission will be indirect cultural transmission with $A$ and $B$ uncorrelated. For direct cultural transmission or indirect transmission with $A$ and $B$ correlated, the $A$ component and $C$ component will also be correlated, which makes a clean variance decomposition impossible. As we will see later, some researchers calculate both $h^{2}$ and $c^{2}$, the second of which is described as the variance 'explained' by common environment. However, such a quantity is only meaningful in the context of indirect cultural transmission with $\operatorname{Cov}(A, B)=0$, and hence is also meaningless when $A$ and $B$ are correlated. 
We have presented some problems with the toy model commonly used in statistical genetics; we do not expect this model to be 'true' or 'realistic'. Simplifications such as random mating, additivity, etc., are unlikely to be the case in reality. However, even in a simple model with direct cultural transmission, if 'heritability'-from what we want it to represent, to its actual definition and estimates-becomes meaningless, how can we expect to have meaningful estimates in empirical research? People might say that such direct cultural transmission is rare, which might reasonably be true for Mendelian phenotypes. However, for social and behavioural traits, this is not likely to be true, and direct cultural transmission may play a very important role, which is in accordance with the general understanding of transmission of phenotypes, and is well known in sociology and anthropology.

Recently, more evidence for the existence of direct cultural transmission has emerged from behavior genetic research. For example, Kong et al. [24] found that parents' non-transmitted alleles were correlated with children's phenotypes. Obviously, a model similar to the above direct cultural transmission model could generate such a signal. Of course, we should be careful in estimating and interpreting such a signal because other demographic mechanisms, such as assortative mating or population subdivision, may produce a similar correlation [23]. It is also important that we distinguish between direct cultural transmission and the phenomenon of Kong et al.'s genetic nurturing. Direct cultural transmission only requires a causal arrow from parents' phenotypes to children's phenotypes, while "genetic nurturing" requires an additional causal arrow from parents' genotypes to parents' phenotypes. This means that genetic nurturing is not a necessary condition for generating the signal, even for direct cultural transmission, since in the correlation between parents' non-transmitted alleles and a child's phenotype, confounding factors between parents' genotypes and phenotypes plus direct cultural transmission, could also generate such a signal. However, if we are able to exclude potential selection biases, such as assortative mating and population subdivision, direct cultural transmission (not necessarily genetic nurturing) can be a reasonable explanation and could be what Kong et al.'s data show. We use "direct cultural transmission" to represent direct phenotypic influence, but it is also possible that the parents' genotypes can influence or be confounded with one parental trait, which has a causal path to another phenotype in children, and will result in a more complex version of parental cultural influence. 
Difficulties in interpreting the correlations between relatives were clearly observed by Wright [2]. He stated (p. 185): 'The point that is sometimes overlooked is that the arrangement for obtaining the best possible prediction equation does not necessarily yield coefficients which have any simple interpretation. ... If child's IQ is affected both by heredity and environment, the same is probably true of parental IQ. Insofar as the latter is determined by environment, it is not a causal factor in relation to child's heredity." Wright goes on to say: "In the case of Miss Burks' data, there is no possible way of distinguishing the effects of environmental factors not included in the measurement of home environment from the contributions of dominance and epistasis or from non-linearity in the combination effects of heredity and environment."

\section{Interpreting Heritability Measures}

As shown above, in the absence of such demographic factors as assortative mating and population subdivision, the widely used estimate of heritability, $2\left(r_{M Z}-r_{D Z}\right)$, turns out to provide an estimate of $h^{2}$ in the presence of direct cultural transmission. $h^{2}$ is neither $H_{\text {narrow }}^{2}$ nor $H_{\text {broad }}^{2}$. In fact, Wright $[1,2]$ and Jensen [4] were aiming to calculate $H_{\text {narrow, }}^{2}$ but were actually calculating $h^{2}$. C and $\mathrm{F}$ showed that with direct cultural transmission, the estimate of broad sense heritability based on the correlation between MZ twins will be systematically biased. As a consequence of C and F, Morton's group updated their 1974 paper [12] and made corrections for assortative mating and direct cultural transmission [15]. They adopted the direct cultural transmission framework and estimated $\operatorname{Var}(A), \operatorname{Var}(B)$, and $\operatorname{Cov}(A, B)$ using minimum chi-squared, and calculated $h^{2}$ and $c^{2}$ correspondingly. Ironically, although they used different methods from Wright and Jensen (minimum chi-squared instead of pedigree analysis), Morton's group also believed they were estimating $H_{\text {narrow, }}^{2}$ but turned out to be estimating $h^{2}$, assuming both cultural transmission and assortative mating. Thus, these analyses mistakenly

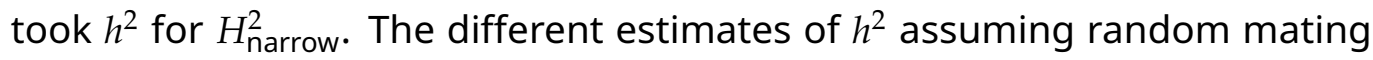
(Wright, Jensen) or assortative mating (Morton's group) were nonetheless theoretically comparable. Thus, from our analysis above we can draw the following conclusions:

1. With direct cultural transmission, neither of these approaches were estimating $H_{\text {narrow, }}^{2}$ although the authors believed they were. 
2. With direct cultural transmission, they were actually estimating $h^{2}$ with or without assortative mating. Thus, the methodological improvement (in estimating $h^{2}$ ) made by Morton's group involved mainly a more careful treatment of assortative mating. Taking direct cultural transmission into account, of course, made their model more realistic, but only made a minor improvement in their estimates. Note that Wright [1,2] and Rao et al. [15] used different data sets, and the difference in their estimates could at least partly be attributed to this. However, here we have focused mainly on the methodology rather than data quality.

3. With direct cultural transmission, as we have pointed out above, because of the correlation between the genetic component and cultural component, $H_{\text {broad }}^{2}, H_{\text {narrow }}^{2} h^{2}$, and the pedigree estimates are all causally meaningless, as is the $c^{2}$ estimated by Rao et al. [15].

\section{Discussion}

In light of these comparisons, how should we evaluate and interpret $\mathrm{C}$ and $\mathrm{F}$ ? What are the importance and limitations of this paper retrospectively? At first glance, the conclusion that the estimate of heritability through MZ twins was inflated seems to be trivial under the ACE model. When $A$ and $C$ are correlated, the $M Z$ covariance is $\operatorname{Var}(A)+\operatorname{Var}(C)+2 \operatorname{Cov}(A, C)$ and is obviously different from $\operatorname{Var}(A)$. However, it is important that although the cultural component in the direct cultural transmission model constitutes the shared environment for twins, it is a special kind of shared environment, one that is common in reality, and intrinsically confounded with the genetic component through the parents' phenotypes. Thus, the inflation of heritability is very difficult to correct in a causally meaningful way. Looking back, it is a pity that $\mathrm{C}$ and $\mathrm{F}$ did not explicitly illustrate these problems, which emerged naturally from their model, but approached them indirectly. Nonetheless, they drew the valid conclusion that, "Side by side with biological transmission, a purely 'cultural' inheritance thus arises, which in the case of parent-offspring interaction is almost completely confounded with biological inheritance." As the first quantitative model to analyze the effect of direct cultural transmission on the estimate of heritability, it provided a minimal framework for analyzing related problems, such as models of genetic nurturing [24].

The problems of defining and extracting the meaning of heritability in the presence of direct cultural transmission have not disappeared in the genomic era. For example, a recent paper incorporated direct cultural transmission into 
the calculation of heritability from GWAS and regressed children's phenotype on children's genotypes and parents' genotypes [25]. Because children's genotypes are correlated with their parents' genotypes, the covariance term may be important. Because the parents' phenotypes are not purely determined by their genotypes, Young et al.'s estimate will not be the $h^{2}$ defined in our recent paper [23], but, both studies face similar problems. In the supplementary material to Young et al. [25], as well as in their previous paper [24], they also aimed at a clean decomposition by regressing the children's transmitted alleles and non-transmitted alleles. However, in this case, the regression coefficient of the transmitted alleles is the joint effect of genetic and direct cultural transmission; it is not purely genetic. Again we face the problem that to produce a clean decomposition, we have to give up trying to distinguish between correlated effects, and, if we want to distinguish between correlated effects, we cannot have a clean decomposition of the total variance.

This problem emerges in other contexts. For example, if a trait is determined by several interacting genes, identifying the contribution of one specific gene to the total phenotypic variance will face a similar problem, which will arise in any system that either involves a generating process of correlated variables, or a system whose variables have non-causal correlation, such as confounding or selection bias, that is difficult to resolve. This difficulty was pointed out by Lewontin [26], who argued that ANOVA was often not a good statistical tool for finding causes. We want to also note that the problem may lie in the quantity we are trying to measure, i.e., "the fraction of variance due to/explained by/caused by ...". A satisfactory counterfactual definition of such a quantity will be elusive unless the variance decomposition is clean.

Although the assessment of $\mathrm{C}$ and $\mathrm{F}$ that we have presented above focuses on the modeling of phenotypic or other types of cultural transmission, it should be remembered that Cavalli-Sforza was also intensely interested in the dynamics of natural selection. In fact, Feldman and Cavalli-Sforza [21] put the framework of $C$ and $F$ and, in the discrete-phenotype case ( $a$ dichotomous phenotype), allowed the fitness of the phenogenotypes to vary. This study may have been the first to formally model gene-culture coevolution under natural selection with cultural transmission. Among the interesting findings from this analysis was that if heterozygotes transmitted an advantageous vertically-transmitted cultural trait more reliably than homozygotes, it does not guarantee a stable polymorphic equilibrium as would be the case if the heterozygote were advantageous in egg-to-adult survival, 
for example. Later, Behar and Feldman [22] extended this model to multiple genetic alleles with results reminiscent of the classical theory of multiple alleles at a single genetic locus.

\section{Competing Interests}

Marcus W. Feldman is a member of the Editorial Board of the journal Human Population Genetics and Genomics. Hao Shen declares that he has no competing interests. The two authors were not involved in the journal's review of or decisions related to this manuscript.

\section{References}

1. Wright S. Statistical methods in biology. J Am Stat Assoc. 1931;26:155-163.

2. Wright S. The method of path coefficients. Ann Math Stat. 1934;5:161-215.

3. Burks BS. The relative influence of nature and nurture upon mental development: a comparative study of foster parent-foster child resemblance and true parent-true child resemblance. In: 27th Yearbook of the National Society for the Study of Education, Part 1. Bloomington, IN: Public School Publishing Co. 1928; 219-316. DOI

4. Jensen A. How much can we boost IQ and scholastic achievement? Harvard Educ Rev. 1969;39:1-123. DOI.

5. Burt C. The genetic determination of differences in intelligence: A study of monozygotic twins reared together and apart. Brit J Psychol. 1966;57:137153. DOI.

6. Kamin LJ. Heredity, intelligence, politics, and psychology (Unpublished). Eastern Psychological Association Convention, May 5, 1973.

7. Kamin LJ. The Science and Politics of IQ. Potomac, Maryland: Lawrence Erlbaum Associates; 1974.

8. Cavalli-Sforza LL, Feldman MW. Cultural versus biological inheritance: Phenotypic transmission from parents to children (A theory of the effect of parental phenotypes on children's phenotypes). Am J Hum Genet. 1973;25:618-637.

9. Fisher RA. The correlation between relatives on the supposition of Mendelian inheritance. Trans Roy Soc Edinburgh. 1918;52:399-433.DOI

10. Feldman MW, Christiansen FB, Otto SP. Gene-culture co-evolution: teaching, learning, and correlations between relatives. Israel J Ecol Evol. 2013;59:72-91. DOI. 
11. Rao DC, Morton NE, Yee S. Analysis of family resemblance. II. A linear model for familial correlation. Am J Hum Genet. 1974;26:331-359.

12. Rao DC, Morton NE, Yee S. Resolution of cultural and biological inheritance by path analysis. Am J Hum Genet. 1976;28:228-242.

13. Cloninger $C R$, Rice J, Reich T. Multifactorial inheritance with cultural transmission and assortative mating. II. A general model of combined polygenic and cultural inheritance. Am J Hum Genet. 1979;31:176-198.

14. Cloninger CR, Rice J, Reich T. Multifactorial inheritance with cultural transmission and assortative mating. III. Family structure and the analysis of separation experiments. Am J Hum Genet. 1979;31:366-388.

15. Rao DC, Morton NE, Lalouel JM, Lew R. Path analysis under generalized assortative mating: II. American IQ. Genet Res. 1982;39:187-198. DOI.

16. Cavalli-Sforza LL, Feldman MW. Evolution of continuous variation: Direct approach through joint distribution of genotypes and phenotypes. Proc Natl Acad Sci USA. 1976;73:1689-1692. DOI.

17. Feldman MW, Cavalli-Sforza LL. The evolution of continuous variation. II. Complex transmission and assortative mating. Theor Pop Biol. 1977;11:161-181. DOI.

18. Feldman MW, Cavalli-Sforza LL. Aspects of variance and covariance analysis with cultural inheritance. Theor Pop Biol. 1979;15:276-307. DOI.

19. Cavalli-Sforza LL, Feldman MW. The evolution of continuous variation. III. Joint transmission of genotype, phenotype and environment. Genetics. 1978;90:391-425. DOI.

20. Otto SP, Christiansen FB, Feldman MW. Genetic and cultural inheritance of continuous traits. Morrison Institute for Population and Resource Studies, Working Paper No. 64, 1995.

21. Feldman MW, Cavalli-Sforza LL. Cultural and biological evolutionary processes, selection for a trait under complex transmission. Theor Pop Biol. 1976;9:239-259. DOI.

22. Behar H, Feldman MW. Gene-culture coevolution under selection. Theor Popul Biol. 2018;121:33-44.

23. Shen H, Feldman MW. Genetic nurturing, missing heritability, and causal analysis in genetic statistics. PNAS. 2020;117:25646-25654. DOI.

24. Kong A, Thorleifsson G, Frigge ML, Vilhjalmsson BJ, Young AI, Thorgeirsson $\mathrm{TE}$, et al. The nature of nurture: Effects of parental genotypes. Science. 2018;359:424-428. DOI. 
25. Young AI, Frigge ML, Gudbjartsson DF, Thorleifsson G, Bjornsdottir G, Sulem $P$, et al. Relatedness disequilibrium regression estimates heritability without environmental bias. Nat Genet. 2018;50:1304-1310. DOI.

26. Lewontin RC. Annotation: the analysis of variance and the analysis of causes. Am J Hum Genet. 1974;26:400-411.

Cite this article: Shen H, Feldman MW. Cultural versus biological inheritance: A retrospective view of Cavalli-Sforza and Feldman (1973). Hum Popul Genet Genom. 2021; 1(1): 0003. https://doi.org/10.47248/hpgg2101010003 